\title{
An Approach to Hierarchical Clustering via Level Surfaces and Convexity
}

\author{
R. Holley, ${ }^{1}$ J. Malitz, ${ }^{1}$ and S. Malitz ${ }^{2}$ \\ ' Department of Mathematics, University of Colorado, \\ Boulder, CO 80309, USA \\ \{holley, malitz\}@euclid.colorado.edu \\ 2 Raytheon Corporation, 16800 CentreTech Parkway, \\ Aurora, CO 80011 , USA \\ smmalitz@west.raytheon.com
}

\begin{abstract}
We consider the following approach to hierarchical data clustering. Let $A=$ $\left\{p_{1}, p_{2}, \ldots, p_{n}\right\}$ be a set of $n$ data points in $R^{d}, d \geq 2$. Define $f(z)=\sum_{i=1}^{n}\left(\left|z-p_{i}\right|_{q}\right)^{-1}$, where $z$ is in $R^{d}$, and $|\cdot|_{q}$ denotes the $L_{q}$-norm, $q \geq 1$. The function $f$ can be viewed as a "combined luminosity" formed by summing individual "point luminosities" located at the $p_{i}$. The level surfaces of $f$ define a hierarchical clustering of $A$ in a natural way. We prove a general result on convexity that enables us to obtain this clustering for $q=1$ by examining the values of $f$ on the edges of a rectilinear grid induced by $A$. An algorithm is developed that is practical in several situations. For $n<1000$, these include: (1) $d$ small (say $d \leq 4$ ) and the $p_{i}$ real; (2) $d$ moderate (say $d \leq 25$ ) and the $p_{i}$ binary.
\end{abstract}

\section{Introduction}

\subsection{Cluster Analysis}

Cluster analysis is a mathematical tool that represents structure in data as groups of elements or as hierarchies of groups. This representation can then be explored to determine if the data fits a preconceived theory of cluster formation, or it can feed into a subsequent decision or test. Central to cluster analysis is the notion of a clustering algorithm which imposes natural structure on data by grouping together elements that are related in a precisely defined way. Two kinds of clustering occur most often in practice: partitional and hierarchical. In partitional clustering, data points are divided into some number of disjoint clusters. In hierarchical clustering, a linear nesting of such partitions is created in which the partition at a given level refines that of the preceding level. Here we are 
concerned with hierarchical clustering. For the interested reader, the book by Jain and Dubes [3] provides an excellent survey of the very large subject of cluster analysis, devoting considerable attention to the pros and cons of algorithms used in practice.

Data elements to be clustered are commonly called patterns. These are formalized in a manner consistent with the desired application. Often patterns are specified as points in a $d$-dimensional Cartesian space, where each axis corresponds to an attribute or feature. As an example, a point $p=\left(x_{1}, x_{2}, \ldots, x_{d}\right)$ might represent, say, a person of height $x_{1}$, body weight $x_{2}$, age $x_{3}$, blood pressure $x_{4}$, etc. Here the $x_{i}$ are real- or integer-valued. In other situations, features may take on binary values, so a pattern $p=0110 \cdots 0$ might represent, say, a person's answers to a yes-no questionnaire, where 1 denotes yes and 0 denotes no. Still other applications require that the value of each attribute be chosen from a finite set of equidistant characters. For example, the character set $\{A, C, T, G\}$ symbolizing the compounds adenine, cytosine, thymine, and guanine, enables one to represent snippets of DNA by patterns of the form GAT ACTTG $\cdots$.

The purpose of this paper is to introduce a new and rather natural approach to hierarchical clustering-one probably best suited for collections of binary patterns or character patterns, but nevertheless well-defined for any pattern set in $R^{d}$. We present an algorithm to implement this approach and analyze its worst-case computational complexity. Admittedly, the analysis shows that in the worst case this algorithm can require significantly more running time and memory than existing algorithms implementing other clustering methods. However, we suspect that its average-case performance is considerably better than its worst case performance, and if not, then other algorithms implementing the approach could be explored. The approach itself has some unique and attractive properties that recommend its usage where it may be feasibly applied.

For example, the great majority of existing hierarchical clustering methods require the definition of an interpattern or intercluster distance function (see the Matrix Updating and Graph Theory Based methods discussed in [3]). When ties occur in this function (a tie being when two or more pattern pairs or cluster pairs register the same distance value) serious ambiguities generally result. Attempting to remedy the problem by making small perturbations in the data so as to break ties often results in clusterings whose structure is highly sensitive to these perturbations-an unpleasant state of affairs. By contrast, our approach does not require an interpattern or intercluster distance function, and so therefore does not suffer such ambiguity problems. Furthermore, using our approach, small perturbations in the data correspond to small changes in the resulting hierarchy, i.e., the clustering generated by our method always varies continuously with the data.

There are some histogram-based partitional clustering algorithms that do not require a distance function (see the Density Estimation and Mode Seeking methods discussed in [3]), but these can only be used practically for low-dimensional pattern spaces. These methods involve decomposing the pattern space into a large number of disjoint rectangular volumes, tallying the number of patterns that fall within each volume, and delimiting clusters along "valleys" in the resulting histogram. Possibly there is a way to extend these methods to generate hierarchical clusterings, but still the memory and run-time requirements of managing high-dimensional histograms would continue to restrict their use to pattern spaces of low dimension. Although we too cannot prove that our approach is suitable for high dimensions, we do know that even in the worst case, our algorithm 
can practically cluster, say, 1000 binary patterns in 25 dimensions. Such a data set would not be practical for the histogram-based method.

Another appealing aspect of our method is that it does not try to impose a binary branching structure on the hierarchical clustering it generates. If the data should possess intrinsic $n$-fold symmetry, where $n>2$, this symmetry will manifest itself in the resulting hierarchy as $n$ distinct clusters merging simultaneously into one. Virtually every other hierarchical clustering algorithm we know about, at a given stage, seeks to merge two existing clusters into one, thereby forcing a binary branching in the hierarchy.

From a purely mathematical perspective, we think our approach is interesting because it relies on an interesting and very classical-sounding result on convexity that we have not seen previously in the literature.

The organization of the paper is as follows. A high-level description of our method is provided in Section 2. The essential convexity result on which it hinges is developed in Section 3. In Section 4 a detailed algorithm for generating a hierarchical clustering based on the ideas of the previous two sections is provided. Section 5 analyzes the worst-case computational complexity of our algorithm, and displays the output of the algorithm for several binary data sets. Section 6 extends our clustering method to handle character data. Section 7 discusses ways to reduce the dimension of the pattern space without losing information. (Reducing dimension improves the running time and memory utilization of our algorithm.) Section 8 closes with a number of open problems.

\section{Method Description}

Consider a set $A=\left\{p_{1}, p_{2}, \ldots, p_{n}\right\}$ of $n$ patterns in $R^{d}$, where each $p_{i}$ is a $d$-vector $\left(p_{i}^{1}, p_{i}^{2}, \ldots, p_{i}^{d}\right)$. A hierarchical clustering of $A$, call it $H C(A)$, can be generated as follows. Imagine placing a point "light source" at each $p_{i}$ where "intensity" or "luminosity" decreases with distance. For each point $z \in R^{d}$, sum the individual luminosities contributed by the $p_{i}$ to form a combined luminosity function $f(z)$. This function can be viewed as a surface over the pattern space $R^{d}$. For any real threshold $t$, the locus of $z$ 's satisfying $f(z) \geq t$ is the union of some minimum number of disjoint connected components $C_{1}, C_{2}, \ldots, C_{k_{t}}$, where the $C_{i}$ 's are delimited by the level surface for $f$ at threshold $t$. The set $A$ is always contained in the union of these $C_{i}$ since $f(z)$ approaches infinity as $z$ approaches any $p_{j}$. Therefore, intersecting $A$ with the $C_{i}$ 's induces a partition of $A$. By varying the threshold $t$ continuously from a large value to a small value, a hierarchical clustering of $A$ is generated. If the largest threshold value is sufficiently large, then each element of $A$ will be isolated in its own cluster; if the smallest threshold is sufficiently small, then all the elements of $A$ will be gathered into one cluster.

A natural question arises: is there an efficient algorithm to generate this hierarchical clustering $H C(A)$ ? For arbitrary point luminosities (i.e., arbitrary in the way luminosity drops off with distance), an obvious algorithm does come to mind, but it is practical for low dimensions only. Similar to the histogram-based approach described in the previous section, the idea is to consider a gradually decreasing sequence of thresholds $t$, and for each such threshold, evaluate $f$ on the lattice points of a "fine" grid in the pattern space as necessary to obtain a good and explicit approximation to the connected components $C_{i}$ defined above. Although such an algorithm is clearly not practical for higher dimensions, 
we were not able to come up with anything provably better. However, as we shall see below, by making a judicious choice of point luminosities, $H C(A)$ can be recovered by evaluating $f$ on a much coarser grid than one would initially expect-this translates into some significant space and run-time gains.

Let $|\cdot|_{q}$ denote the $L_{q}$ norm, $q \geq 1$. Let $h(y)$ be a continuous decreasing convex function over the domain $(0, \infty)$ that tends to infinity as $y$ tends to 0 from above. We call $h$ an attenuation function. Natural examples include $y^{-\alpha}$ where $\alpha \in(0, \infty)$, and $-\log y$. Let us say that a point light source at the origin in $R^{d}$ is an $L_{q}$ light source if the luminosity at a point $z$ in $R^{d}$ equals $h\left(|z|_{q}\right)$ for some $h$ as above. Let $H C(A ; q, h)$ denote the hierarchical clustering of $A$ as defined in the first paragraph of this section using $L_{q}$ light sources at the data points and attenuation function $h$.

At present, we know of no provably faster algorithm to generate $H C(A ; q, h)$ for $q>1$ than the algorithm described above which evaluates $f$ on the lattice points of a "fine" grid in the pattern space. For $q=1$, however, we shall show that all necessary information about $f$ can be garnered from its values on the edges of a "coarse" rectilinear grid, $G(A)$, induced by the elements of $A$. The grid points of $G(A)$ are the elements of the Cartesian product

$$
\left\{p_{1}^{1}, \ldots, p_{n}^{1}\right\} \times\left\{p_{1}^{2}, \ldots, p_{n}^{2}\right\} \times \cdots \times\left\{p_{1}^{d}, \ldots, p_{n}^{d}\right\}
$$

Clearly, if there are $n$ data points in general position in $R^{d}$, the number of edges in the grid $G(A)$ grows like $O\left(d n^{d}\right)$. In the worst case, our algorithm will explore all these edges. For $n$ patterns in $R^{d}$ with $n<1000$, this may be feasible only for small $d$, say $d \leq 4$. However, if the patterns are binary, the number of edges in the grid $G(A)$ grows like $O\left(d 2^{d}\right)$, and it is practical to explore this many edges for, say $d \leq 25$, with today's chip speeds and RAM capacities. As for the average-case performance of our algorithm (which may be much better than the worst case) we know of no nontrivial bound, but we have been surprised to see how fast our computer program runs on various examples in dimensions as high as 25 . For these examples at least, remarkably few edges of the hypercube were explored.

We can briefly state how we are able to reduce the problem of generating $H C(A ; 1, h)$ to the problem of evaluating $f$ on the edges of the grid $G(A)$-a detailed argument appears in the next section. For any real threshold $t$, two patterns $p_{i}, p_{j}$ in data set $A$ belong to the same connected component of $\{z: f(z) \geq t\}$ iff there is a path $P$ in $R^{d}$ from $p_{i}$ to $p_{j}$ such that $f(w) \geq t$ for every $w$ along $P$. In the special case of $L_{1}$ light sources, it is easy to show that the combined luminosity function $f$ is convex throughout each cell of the grid $G(A)$. Under this condition, it can be shown that there is a path $P$ in $R^{d}$ from $p_{i}$ to $p_{j}$ with $f(w) \geq t$ for every $w$ along $P$ iff there is a grid-path $Q$ in the grid $G(A)$ from $p_{i}$ to $p_{j}$ with $f(w) \geq t$ for every $w$ along $Q$. This completes the reduction.

The clustering $H C(A ; 1, h)$ is readily obtained once we know for each pair of data points $p_{i}, p_{j}$, the maximum value of $t$ such that $p_{i}$ and $p_{j}$ are in the same connected component of $\{z: f(z) \geq t\}$. As observed above, this problem reduces to finding a "highest" path in the grid $G(A)$ between $p_{i}$ and $p_{j}$. In fact, there is actually a tree inside $G(A)$ that spans all the data points and contains a "highest" grid path between each pair of data points. Essentially what our algorithm does is generate this tree in a systematic way. 


\section{Convex Bodies, Convex Functions, and Highest Paths}

Let $B$ be a closed bounded convex body in $R^{d}$ and let $f$ be a convex function defined throughout $B$. A $B$-path between two points $u, v \in B$ is a continuous curve from $u$ to $v$ that lies wholly within $B$. The altitude of such a $B$-path is the minimum $f$-value assumed anywhere along its trajectory. We say that a $B$-path is a highest $B$-path from $u$ to $v$ if its altitude is no less than that of any other $B$-path from $u$ to $v$. Given two $B$-paths $P_{1}, P_{2}$ (possibly connecting different endpoints), we say that $P_{1}$ is higher than $P_{2}$ if $P_{1}$ 's altitude is greater than $P_{2}$ 's altitude.

Theorem 3.1. Let $B$ be a closed bounded convex body in $R^{d}(d \geq 2)$ and let $f$ be a convex function defined throughout $B$. Let $s$ and $t$ be two points on the boundary $\partial B$ of $B$. Then there is a highest $B$-path from $s$ to $t$ that lies wholly within $\partial B$.

Proof. Let $P$ be a highest $B$-path from $s$ to $t$, and let $m$ be the minimum $f$-value achieved along $P$. Let $A$ be the open subset of $B$ that consists of all points with an $f$-value strictly less than $m$.

If $A$ is empty, then $m$ is the minimum $f$-value assumed anywhere in $B$. This means that an arbitrary path $Q$ from $s$ to $t$ lying wholly within $\partial B$ does not assume a value less than $m$ anywhere along its trajectory. Therefore, $Q$ is at least as high as the highest path $P$.

If $A$ is not empty, then $A$ is a convex set by the convexity of $f$. Pick an arbitrary point $O$ inside $A$. Thinking of $O$ as a point light source, project $P$ onto the boundary $\partial B$ of $B$, and let $Q$ denote the resulting shadow path. Not only does $Q$ lie entirely within $\partial B$, but it also avoids $A$. For suppose there were a point $q \in Q \cap A$. Since $q$ is an element of the shadow path $Q$, there is a point $p \in P$ such that $O, p$, and $q$ all lie in a straight line with $p$ in the middle. However, $O$ and $q$ both belong to $A$ while $p$ does not, and this violates the convexity of $A$. Thus $Q$ avoids $A$, which means $Q$ is at least as high as $P$.

The following lemma is routine and the proof is omitted.

Lemma 3.1. Let $B$ be a closed bounded convex polyhedron in $R^{d}$ and let $f$ be a convex function defined throughout $B$. Let $s$ be any point of $B$. Then there is a vertex $v$ of $B$ such that along the line segment from $s$ to $v$ the value of $f$ does not drop below $f(s)$.

Theorem 3.2. Let $B$ be a closed bounded convex polyhedron in $R^{d}(d \geq 2)$ and let $f$ be a convex function defined throughout $B$. Let $s$ and $t$ be two vertices of $B$. Then there is a path of edges from $s$ to $t$ that is a highest $B$-path from $s$ to $t$.

Proof. The proof is by induction on dimension $d$ of $B$.

If $d=2$, the result holds by Theorem 3.1 above.

Suppose the theorem holds for all dimensions $k$, where $2 \leq k<d$. By Theorem 3.1 above, there is a highest $B$-path $P$ from $s$ to $t$ that lies entirely within $\partial B$. Let $F_{1}, F_{2}, \ldots, F_{n}$ be the sequence of $(d-1)$-faces traversed by $P$ in going from $s$ to $t$. Thus 
$P$ can be chopped into closed subpaths $P_{1}, P_{2}, \ldots, P_{n}$ such that $P_{i}$ is contained within $F_{i}$ for each $i$. Let $v_{i-1}$ and $v_{i}$ be the endpoints of $P_{i}$ where $v_{0}=s$ and $v_{n}=t$. Notice that $v_{i}$ lies within $F_{i} \cap F_{i+1}$ which is a convex polyhedron $C$. By Lemma 3.1 above, there is a vertex $u$ of $C$ (which is necessarily a vertex of both $F_{i}$ and $F_{i+1}$ ) such that along the line segment from $v_{i}$ to $u$ the value of $f$ does not drop below $f\left(v_{i}\right)$. Notice also that this line segment is contained in $C$, the shared boundary of $F_{i}$ and $F_{i+1}$. It follows that there is a path $Q$ from $s$ to $t$ consisting of closed subpaths $Q_{1}, Q_{2}, \ldots, Q_{n}$ in tandem such that each $Q_{i}$ is contained in $F_{i}$, the endpoints of each $Q_{i}$ are vertices of $F_{i}$, and each $Q_{i}$ is at least as high as $P_{i}$. By the induction hypothesis, each path $Q_{i}$ can be replaced by a path $R_{i}$ consisting of edges of $F_{i}$ (and thus edges of $B$ ) that is at least as high. The path $R$ consisting of $R_{1}, R_{2}, \ldots, R_{n}$ in tandem is the desired $B$-path.

Theorem 3.3. Let $K$ be a connected complex in $R^{d}(d \geq 2)$ of d-dimensional closed bounded convex polyhedra $B_{1}, B_{2}, \ldots, B_{q}$. Let $f$ be a continuous function defined throughout $K$ such that the restriction of $f$ to each $B_{i}$ is convex. Let $s$ be a vertex of $B_{i}$ and let $t$ be a vertex of $B_{j}$. Then there is an edge-path in $K$ from $s$ to $t$ that is a highest path within $K$ from s to $t$.

Proof. The proof is almost identical to that of Theorem 3.2.

Theorem 3.4. The combined luminosity function $f(z)$, obtained by summing $L_{1}$ point luminosities located at the patterns $p_{i}$ in $A$, is convex over each closed cell of the grid $G(A)$. Thus (by Theorem 3.3) for any pair of data points $p_{i}, p_{j}$ in A there is a path of grid edges in $G(A)$ from $p_{i}$ to $p_{j}$ that is a highest path in $R^{d}$ from $p_{i}$ to $p_{j}$.

Proof. Let $f_{1}, f_{2}, \ldots, f_{n}$ be the $L_{1}$ point luminosities associated with the patterns $p_{1}, p_{2}, \ldots, p_{n}$ in $A$, respectively. Let $B$ be any closed cell of the grid $G(A)$. If we show that each $f_{i}$ is convex over $B$, then the theorem follows since the sum of convex functions is convex.

It suffices to show that $f_{i}$ is convex over each orthant about $p_{i}$. By definition, $f_{i}(z-$ $\left.p_{i}\right)=h\left(\left|z-p_{i}\right|_{1}\right)$ where $h$ is a convex decreasing function. Without belaboring the point, $f_{i}$ is readily seen to be convex over any orthant about $p_{i}$ by observing that it is the composition of a convex decreasing function $h$ with a linear function $|\cdot|_{1}$.

\section{Algorithm to Generate $\mathrm{HC}(A ; 1, h)$}

Let $G=(V, E)$ be an arbitrary graph and let $w: E \rightarrow R$ be a function that assigns real-valued weights to the edges of $G$. Let $s$ and $t$ be two vertices of $G$. The altitude of a simple path $P$ in $G$ connecting $s$ and $t$ is the weight of the minimum-weight edge belonging to $P$. Say $P$ is a highest path from $s$ to $t$ if no other path in $G$ from $s$ to $t$ has greater altitude.

Let $T$ be a spanning tree for $G$. We say $T$ is a maximum-weight spanning tree (MST) if the sum of its edge-weights is no less than that of any other spanning tree for $G$.

Let $A$ be a subset of $V$, and let $T$ be a tree in $G$ that spans $A$. Call $T$ a tree of highest 
paths spanning $A$ if for every pair of vertices $s, t$ in $A$, the path from $s$ to $t$ in $T$ is a highest path from $s$ to $t$ in $G$. The following result is well known.

Theorem 4.1. Let $G=(V, E)$ be a graph and let $w: E \rightarrow R$ be a real-valued weight function on the edges of $G$. Let $A$ be a subset of $V$, and let $T$ be a tree in $G$ that spans $A$. Then $T$ is a tree of highest paths for $A$ iff $T$ is a subtree of some MST for $G$.

The following discussion forms the basis of our computer program to generate the hierarchical clustering $H C(A ; 1, h)$. Thus far we have considered the grid $G(A)$ induced by pattern set $A$ as a geometric object with points, line segments, and cells. We now wish to view it as a simple undirected graph. Let $G(A)=\left(V_{A}, E_{A}\right)$ where $V_{A}$ is the set of grid points induced by $A$, and $E_{A}$ consists of grid-point pairs that are directly connected by a grid line segment (i.e., no third grid point lies on the line segment connecting the two grid points). In addition, define a real-valued weight function $w$ on $E_{A}$ such that $w((u, v))$ equals the minimum value of $f$ attained along the line segment from $u$ to $v$ in the geometric realization of $G(A)$. (Note that $f$ is convex along each line segment of $G(A)$, and the convex minimization problem in one dimension has fast algorithms. See [4].) We know that $H C(A ; 1, h)$ can be inferred from a tree (in graph $G(A)$ ) of highest paths for $A$, and we know by Theorem 4.1 that a tree of highest paths for $A$ is a subtree of an MST for $G(A)$. Thus our goal is to generate an MST spanning $A$. To that end we employ the greedy MST algorithm of Prim (see [2]). For our application, we can terminate Prim's algorithm at the moment the last point of $A$ is added to the existing Prim tree rather than explore all the edges of $G(A)$.

Now the Prim tree $T$ (tree of highest paths for $A$ ) generally has far more vertices than $A$ has patterns. Furthermore, its topology does not mimic the hierarchical clustering $H C(A ; 1, h)$ in a transparent way. To obtain $H C(A ; 1, h)$ from the Prim tree directly is therefore a little cumbersome. Thus we invoke the remark below (proof omitted) to generate a secondary tree whose vertices are exactly $A$ and whose topology mimics $H C(A ; 1, h)$ exactly.

Remark 4.1. Let $T=(V, E)$ be a tree, and let $w: E \rightarrow R$ be a real-valued weight function on the edges of $T$. Let $A$ be a subset of $V$. Then in time $O(|V|)$ it is possible to construct a tree $T^{*}=(A, F)$ such that for every pair of vertices $u, v \in A$, the altitude of the path in $T^{*}$ from $u$ to $v$ is the same as that of the path in $T$ from $u$ to $v$.

We now generate $H C(A ; 1, h)$ from $T^{*}$ in time $O(|A|)$ iteratively as follows. Sort the edges of $T^{*}$ by decreasing weight: $w\left(e_{1}\right) \geq w\left(e_{2}\right) \geq \cdots \geq w\left(e_{n-1}\right)$. Initially, each data point is in its own cluster. At iteration $i$, merge all pairs of clusters that are spanned by an edge of $T^{*}$ with weight less than or equal to the threshold $w\left(e_{i}\right)$. Repeat until all the data points are gathered into one cluster.

\section{Algorithmic Efficiency and Some Examples}

In our implementation of the algorithm described above, we maintain three primary data structures: a priority queue (implemented as a binary tree heap) for the visited vertices of $G(A)$ not currently participating in the Prim tree; a dictionary (implemented as a red- 
black tree) of all the visited vertices of $G(A)$; and a tree data structure that represents the Prim tree itself.

A careful analysis would show that the running time of our implementation is $O$ ( $p$ $(n, d) N)$ where $p(n, d)$ is a low-degree polynomial in $n$ and $d$, and $N$ is the number of vertices of $G(A)$ explored by the algorithm. For data points in $R^{d}$ in general position, the number of vertices in $G(A)$ is $n^{d}$, and this is an upper bound on $N$. Hence, in the worst case, the running time of the algorithm is $O\left(p(n, d) n^{d}\right)$.

The amount of space needed by our implementation is proportional to the number of vertices explored. Hence the worst-case space utilization is $O\left(n^{d}\right)$.

For binary data, the situation is better, though still exponential. In this case the number of vertices in $G(A)$ is $2^{d}$. Hence, the worst-case running time is $O\left(p(n, d) 2^{d}\right)$, and the worst-case space requirement is $O\left(2^{d}\right)$. The algorithm actually achieves this worstcase performance in the situation where, for example, $\boldsymbol{A}$ consists of two vertices at diametrically opposite corners of the binary $d$-cube.

In general though, worst-case performance says little about average-case performance. Even though our algorithm is worst-case exponential, there is still the possibility that its average-case performance is polynomial or at least considerably faster than worst case. (In measuring average-case performance, data sets $A$ of a given cardinality would be selected from the binary $d$-cube in some random way.) We have been surprised to see how fast our computer program runs on various examples in dimensions as high as 25-for these examples, remarkably few edges of the hypercube were explored. Perhaps some other algorithm can be developed which provably computes an MST spanning $A$ in worst-case polynomial time. We leave this as an open question.

In the examples that follow, we ran our algorithm against various binary data sets and displayed the results via tree-like structures called dendrograms. Dendrograms represent hierarchical clusterings in an obvious way and show the relative thresholds at which clusters merge. In all the examples that follow, we have arbitrarily chosen to use attenuation function $h(y)=1 / y$. Our program was run on a Sun SPARCstation 10 and completed each example in a second or less.

\subsection{Example 1}

Consider a "line" of equally spaced points in the ten-dimensional hypercube. The 11 points along the line are of the form $1^{i} 0^{j}$ where $0 \leq i \leq 10$ and $j=10-i$. Our computer program generated the dendrogram shown in Fig. 1. At each level of the hierarchy, three clusters are merged: a symmetric central cluster that contains points from the middle of the line, and two singleton clusters, one on either side of the central cluster. If one interprets this dendrogram as describing an evolutionary history of the data, then a progenitor first gave rise to the line's endpoints, and later to points near the middle.

\subsection{Example 2}

Consider two "solid spheres" of Hamming radius 2 centered at diagonally opposite points $0^{6}$ and $1^{6}$ of the six-dimensional hypercube. Figure 2 shows the clustering generated by 


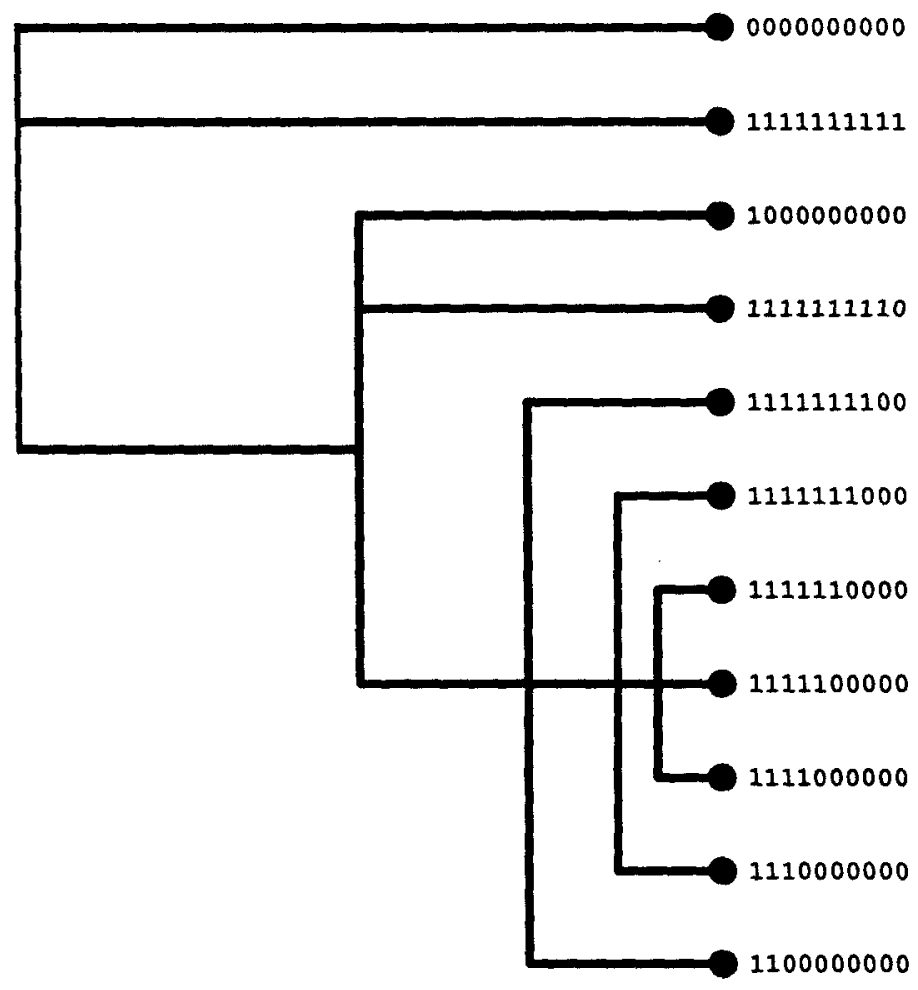

Fig. 1. Generated dendrogram for Example 1.

our program. Apparent in the dendrogram are the $n$-fold symmetries of the data for $n>2$.

\subsection{Example 3}

Here we consider a more general arrangement of data. We contrive three groups $A, B, C$, where $A$ consists of three subgroups of three data points each in a symmetric ensemble, $B$ consists of two subgroups, one with three data points, the other with two, and $C$ consists of a group with only one data point. We isolated the groups from one another so that the group-structure would be apparent. Our program generated the dendrogram shown in Fig. 3. The generated hierarchy recovers the structure we contrived into our data.

\section{Extension to Character Data}

For some clustering applications, the $d$-dimensional pattern set $A=\left\{p_{1}, p_{2}, \ldots, p_{n}\right\}$ is such that the $i$ th feature in each pattern is chosen from a finite set $\Sigma^{i}=\left\{\sigma_{1}^{i}, \sigma_{2}^{i}, \ldots, \sigma_{c_{i}}^{i}\right\}$ of pairwise equidistant characters. Binary patterns, for example, fit exactly into this 


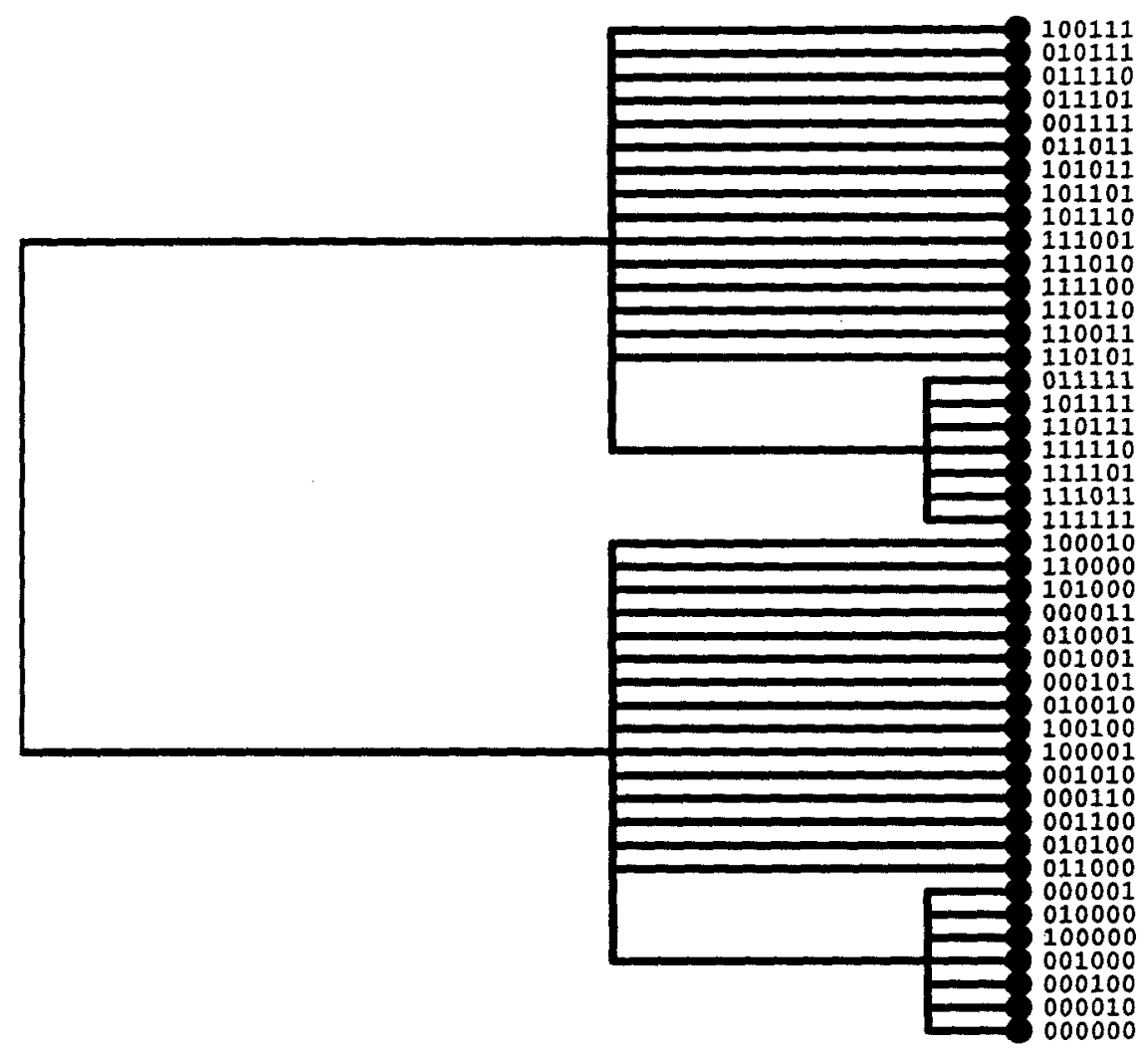

Fig. 2. Generated dendrogram for Example 2.

model, where the cardinality of each $\Sigma^{i}$ equals 2 . We wish to extend our clustering method to handle character data where the cardinality of each $\Sigma^{i}$ can be greater than 2 . The idea is to proceed by analogy with the binary case. That is, first construct a natural convex polyhedron $P$ whose vertices contain the pattern set $A$; then introduce natural convex point luminosities at the vertices of $P$ that belong to $A$.

For each character set $\Sigma^{i}$, of cardinality $c_{i}$, create the $c_{i}$-dimensional simplex $S^{i}$ in $R^{c_{i}}$ whose vertices are the standard unit vectors indexed by the characters in $\Sigma^{i}$. If we let $P$ be the Cartesian product $S^{1} \times S^{2} \times \cdots \times S^{d}$, then $P$ is a convex polyhedron whose vertices contain the pattern set $A$.

For any fixed vertex $\sigma_{j}^{i}$ of simplex $S^{i}$, and any point $z^{i}=\left(z_{1}^{i}, z_{2}^{i}, \ldots, z_{c_{i}}^{i}\right)$ within the volume of this simplex, define the function $\delta\left(\sigma_{j}^{i}, z^{i}\right)=1-z_{j}^{i}$. Observe that this function is linear in $z^{i}$, equals 0 when $z^{i}=\sigma_{j}^{i}$, and equals 1 when $z^{i}=\sigma_{k}^{i}$ for $k \neq j$. Now define the $L_{1}$-distance between a pattern $p_{j}=\left(p_{j}^{1}, \ldots, p_{j}^{d}\right)$ at a vertex of $P$ and a point $z=\left(z^{1}, \ldots, z^{d}\right)$ within the volume of $P$ to be

$$
\delta_{1}\left(p_{j}, z\right)=\sum_{i=1}^{d} \delta\left(p_{j}^{i}, z^{i}\right)
$$




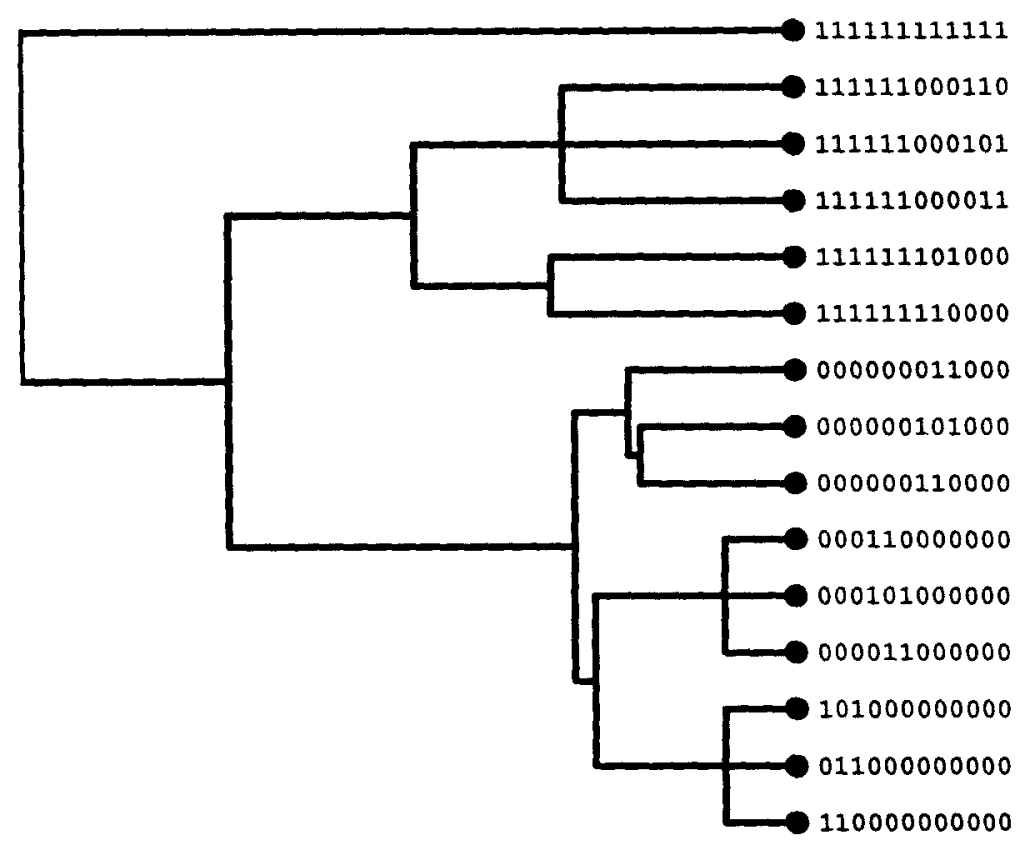

Fig. 3. Generated dendrogram for Example 3.

where $p_{j}^{i}$ is a vertex of simplex $S^{i}$, and $z^{i}$ is the component of $z$ that lies within the volume of $S^{i}$. Observe that for a fixed pattern $p_{j}$, this distance is a linear function in $z$ since it is the sum of linear functions. Now consider a point light source at pattern $p_{j}$ whose luminosity is given by $h\left(\delta_{1}\left(p_{j}, z\right)\right)$ where $h$ is a continuous convex decreasing function over the domain $(0, \infty)$. An $h$ with these properties composed with a linear function yields a luminosity function that is convex throughout the polyhedron $P$.

The stage is now fully set for character data. The hierarchical clustering $H C(A ; 1, h)$ can be found by examining the value of the combined luminosity function on the edges of the polyhedron $P$. The number of vertices in the polyhedron $P$ is equal to the product $\Pi_{i=1}^{d} c_{i}$ of character-set cardinalities, and the number of edges in $P$ is $\left(\sum_{i=1}^{d}\right.$ $\left.\left(c_{i}-1\right)\right)\left(\Pi_{i=1}^{d} c_{i}\right)$. Hence the worst-case running time of the method is $O\left(\left(\sum_{i=1}^{d}\left(c_{i}-\right.\right.\right.$ 1)) $\left.\left(\Pi_{i=1}^{d} c_{i}\right)\right)$ and the worst-case memory requirements are $O\left(\Pi_{i=1}^{d} c_{i}\right)$.

\section{Reducing Problem Dimension}

The worst-case complexity of our algorithm depends most significantly on the dimension of the pattern space, a dependence that is exponential in nature. It therefore makes sense to preprocess the data beforehand to try and reduce the problem dimension as much as possible while preserving inter-pattern $L_{i}$ distances. Here we mention three ways to do this. Again let $A=\left\{p_{1}, p_{2}, \ldots, p_{n}\right\}$ be a pattern set of dimension $d$. Let $p_{j}^{i}$ denote the $i$ th feature of pattern $p_{j}$. Let $\Sigma^{i}$ be the character set for feature $i$. 
Omit Redundant Characters and Features. Let $\Lambda^{i}$ be the subset of $\Sigma^{i}$ each of whose characters actually appear as the $i$ th feature of some pattern in $A$. If $\left|\Lambda^{i}\right|<\left|\Sigma^{i}\right|$, then replace $\Sigma^{i}$ by $\Lambda^{i}$. If $\left|\Lambda^{i}\right|=1$, then omit dimension $i$ altogether.

Coalesce Dimensions with Identical Projection. Let $S \subseteq\{1,2, \ldots, d\}$ be a subset of pattern space dimensions such that for all $i, j \in S$, there holds $p_{k}^{i}=p_{k}^{j}$ for all patterns $p_{k}$ in $A$. Let $m$ be the cardinality of $S$. If $m \geq 2$, then coalesce the dimensions of $S$ into a single dimension, and multiply the $L_{1}$ distance contribution of this new dimension by the weighting factor $m$.

Exploit Isometries of $L_{1}$. The following theorem due to Assouad [1] may on occasion prove useful for reducing problem dimension.

Theorem 7.1. Let $M=\left(X,|\cdot|_{1}\right)$ be an n-element metric subspace of $\left(R^{d},|\cdot|_{1}\right)$. Let $k=\left(\begin{array}{l}n \\ 2\end{array}\right)$. Then there is an isometric embedding $\varphi$ taking $M$ into $\left(R^{k},\left.1 \cdot\right|_{1}\right)$.

This theorem is of interest for our purposes when $k<d$. The isometric embedding $\varphi$ can be obtained from a linear program. This $\varphi$ has the property that if the elements of $M$ lie at the corners of a binary cube in dimension $d$, then their images under $\varphi$ lie at the corners of a rectangular parallelepiped in dimension $k$. An analogous statement can be made for character pattern spaces.

\section{Open Problems}

The following are some open questions concerning the present work.

1. Is there a polynomial-time algorithm for the following problem: given a set $A$ of $n$ binary data points in $\{0,1\}^{d}$, each an $L_{1}$ light source, two points $u, v \in A$, and a rational value $k$, decides whether or not there is a path in the $d$-dimensional unit cube with altitude greater than $k$ ?

2. What is the average-case running time of our algorithm as a function of the number $n$ of patterns and the dimension $d$ of the pattern space? It is intended that the average be taken over the space of "randomly generated" pattern sets where "randomly generated" must be suitably defined. On various examples in dimensions as high as 25 , we have noticed that our algorithm generates the hierarchical clustering $H C(A ; 1, h)$ in a surprisingly short amount of time.

3. Is there a randomized polynomial-time algorithm that computes $H C(A ; 1, h)$ ?

4. Can parallelism be exploited in a useful way in the computation of $H C(A ; 1, h)$ ?

5. What can be said about the relationship between hierarchical clusterings $H C(A$; $\left.1, h_{1}\right)$ and $H C\left(A ; 1, h_{2}\right)$ where $h_{1}$ and $h_{2}$ are different attenuation functions? What can be said about the relationship between hierarchical clusterings $H C(A ; 1, h)$ and $H C(A ; 2, h)$ ?

6. Is there a provably more efficient algorithm for generating $H C(A ; 2, h)$ than the algorithm described earlier based on evaluating the combined luminosity function at the lattice points of a "fine" grid in the pattern space? 


\section{Acknowledgment}

We thank an anonymous referee for suggesting some simplifications to our original exposition, specifically on the extension of our approach to character data.

\section{References}

1. Assouad, P., Deza, M. (1980), Metric Subspaces of $L_{1}$, Publications Mathematiques D'Orsay, Universite de Paris-Sud, Orsay.

2. Cormen, T.H., Leiserson, C.E, Rivest, R.L. (1990), Introduction to Algorithms (Cambridge, MA: The MIT Press).

3. Jain, A.K., Dubes, R.C. (1988), Algorithms for Clustering Data (Englewood Cliffs, NJ: Prentice Hall).

4. Press, W.H., Teukolsky, S.A., Vetterling, W.T., Flannery, B.P., (1992), Numerical Recipes in C: The Art of Scientific Computing (New York: Cambridge University Press).

Received March 10, 1999, and in revised form June 21, 2000. Online publication January 17, 2001. 